\title{
MRI of neuronal network structure, function, and plasticity
}

\author{
Henning U. Voss ${ }^{1, *}$ and Nicholas D. Schiff ${ }^{2}$ \\ ${ }^{1}$ Citigroup Biomedical Imaging Center, Weill Cornell Medical College, New York, NY, USA \\ ${ }^{2}$ Department of Neurology and Neuroscience, Weill Cornell Medical College, New York, NY, USA
}

\begin{abstract}
We review two complementary MRI imaging modalities to characterize structure and function of neuronal networks in the human brain, and their application to subjects with severe brain injury.

The structural imaging modality, diffusion tensor imaging, is based on imaging the diffusion of water protons in the brain parenchyma. From the diffusion tensor, several quantities characterizing fiber structure in the brain can be derived. The principal direction of the diffusion tensor has been found to depend on the fiber direction of myelinated axons. It can be used for white matter fiber tracking. The anisotropy (or directional dependence) of diffusion has been shown to be sensitive to developmental as well as white matter changes during training and recovery from brain injury.

The functional MRI imaging modality, resting state fMRI, concerns the functional connectivity of neuronal networks rather than their anatomical structure. Subjects undergo a conventional fMRI imaging protocol without performing specific tasks. Various resting state network patterns can be computed by algorithms that reveal correlations in the fMRI signal. Often, thalamic structures are involved, suggesting that resting state fMRI could reflect global brain network functionality. Clinical applications of resting state fMRI have been reported, in particular relating signal abnormalities to neurodegenerative processes. To better understand to which degree resting state patterns reflect neuronal network function, we are comparing network patterns of normal subjects with those having severe brain lesions in a small pilot study.
\end{abstract}

Keywords: MRI; neuronal networks; diffusion tensor imaging (DTI); functional MRI (fMRI); resting state; brain injury; axonal regrowth

\section{Introduction}

The impressive advancement of magnetic resonance imaging (MRI) in the past three decades provides us with sensitive tools to obtain

\footnotetext{
*Corresponding author.

Tel.: +1 212746 5216; Fax: +1 212746 6681;

E-mail: hev2006@med.cornell.edu
}

noninvasive, unprecedented characterizations of the human brain in vivo. As highly versatile machines that can be programmed to yield an ever-expanding variety of different image contrasts, MRI scanners now allow us to ask specific questions about structural and functional connectivity of the brain. In this contribution, we review two complementary MRI imaging modalities with a potential to characterize therapeutic response or recovery after brain injury. 
The first imaging modality, diffusion tensor imaging (DTI), is based on imaging the strength and direction of thermal diffusion of water protons in the brain parenchyma. From the diffusion tensor, several quantities can be derived that indirectly characterize fiber structure in the brain, particularly in the white matter. Direction of diffusion has been found to depend on the predominant fiber direction of myelinated axons and can be used for white matter fiber tracking. Here we review applications of DTI based on the anisotropy (or directional dependence) and strength of diffusion, with emphasis on quantitative measures that sensitively reflect white matter changes during recovery from brain injury, as well as during development.

The second imaging modality, resting state fMRI, is based on the blood-oxygen-level-dependent (BOLD) effect in gray matter during rest and concerns the functional connectivity of neuronal networks rather than their anatomical structure. In resting state fMRI, subjects undergo a conventional fMRI imaging protocol without performing specific tasks. The resulting signal contains various resting state network patterns, which are obtained by algorithms that reveal correlations in the signal. The neurophysiological origins of resting state BOLD fluctuations are not completely understood; neither is the potential use of this measurement for imaging therapeutic response nor its recovery after brain injury. However, during the past 3 years, several clinical applications of resting state fMRI have been reported. Here we review, in particular, applications that relate signal abnormalities to neurodegenerative processes. In addition, we explore the possibility to use this assessment of network integrity in patients with brain injuries. We describe a pilot study with two patients with multiple focal brain lesions, and discuss the potential of resting state fMRI as a tool to assess disruptions and recovery of neuronal networks after brain injury.

Both DTI and resting state fMRI are reviewed with most recent applications to brain injury. Rather than providing a comprehensive review over the techniques, we will focus on the future potential of these techniques as clinical research tools.

\section{Diffusion tensor imaging}

\section{Technique and applications}

DTI is an imaging modality capable of rendering the local direction of fibers and characterizing microscopic tissue properties (Basser et al., 1994; Coremans et al., 1994; Basser and Pierpaoli, 1996). Particularly in the brain parenchyma, diffusion of water parallel to myelinated white matter is larger than that perpendicular to the fiber tracts, where diffusion is hindered by myelin sheaths (Beaulieu and Allen, 1994). Thus, diffusion of water in white matter depends on the direction, i.e., it is anisotropic. In contrast, diffusion in a glass of water or in the cerebrospinal fluid is largely direction-independent, i.e., isotropic. The diffusion constant and other parameters describing diffusion depend on microscopic tissue properties such as density, diameter, degree of myelination, and the geometry of fibers, and influence the MRI signal (LeBihan et al., 1986; Douek et al., 1991; Basser, 1995; Alexander et al., 2007; Assaf and Pasternak, 2008; Mukherjee et al., 2008a). The apparent diffusion of water ("apparent" since physiological processes that are not strictly diffusive in a physical sense may contribute; Neil, 1997) is described, in particular, by the apparent diffusion coefficient (ADC), the degree of anisotropy (e.g., expressed by the fractional anisotropy, FA) and the predominant direction of diffusion. A sketch of the DTI methodology and definitions of these quantities is provided in Fig. 1.

Recently, DTI has attracted much interest in both clinical neurology and basic neurobiological research for its ability to render white matter fiber structures, which cannot be seen in conventional magnetic resonance imaging. For these "fiber tracking" methods, see, for example, Basser (1995); Mori and van Zijl (2002); Mori et al. (2005). The accuracy of DTI-based fiber tracts compared to true neuronal fiber tracts has been continuously improved to the point where, for some structures, DTI fiber tracking approaches or even outperforms the accuracy of anatomical atlases (Douek et al., 1991; Holodny et al., 2005; Ino et al., 2007; Kim et al., 2008; Park et al., 2008). 

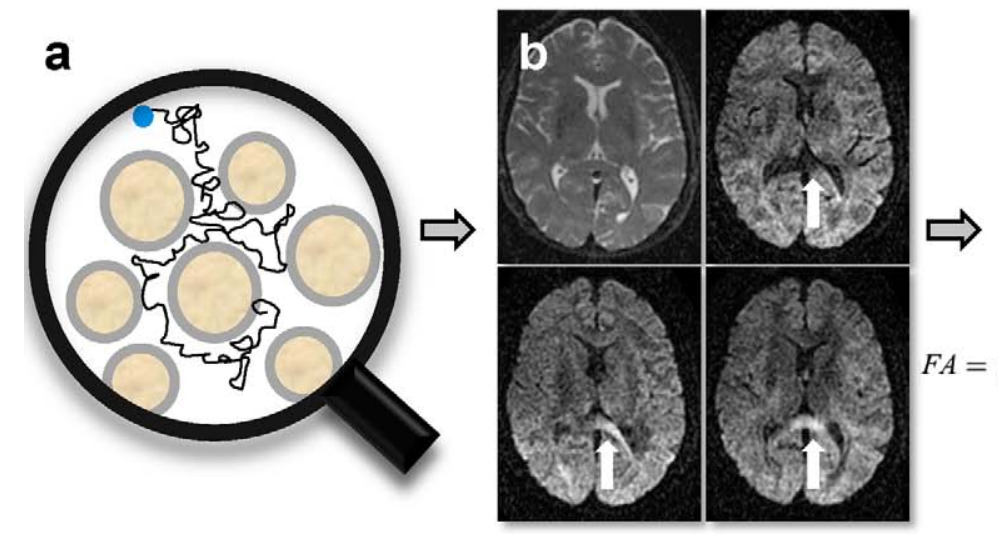

C
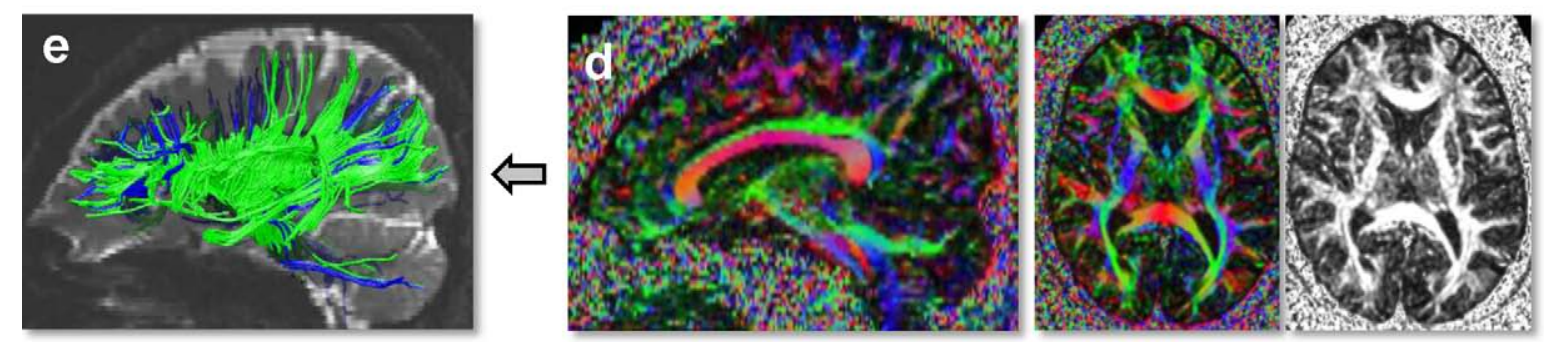

Fig. 1. From microscopic diffusion to cerebral fiber tract reconstructions. (a) Microscopic thermal diffusion of water protons in the interstitial space between myelinated axons is not isotropic but restricted in the directions perpendicular to the fibers. It is less restricted in the direction parallel to the fibers, which would be out-of-plane here. Schematically sketched is a possible diffusive trajectory of a water proton. There is also diffusion of water within the fibers and across the myelin sheaths, which provides a usually smaller contribution to the average diffusion properties of the tissue. (b) A T2 weighted image and three representative axial MRI diffusion weighted images of the brain of a normal subject. The three diffusion weighted images have been acquired with diffusion gradients probing diffusion along different directions. It is evident how the different gradient directions determine image intensity, for example in the splenium of the corpus callosum (arrows). (c) From the T2 and diffusion weighted images, for each voxel a series of mathematical operations is performed. First, a diffusion tensor D is computed by an overdetermined least squares algorithm. In this case, one T2 weighted and 55 diffusion weighted images have been acquired, from which the six independent components of D were estimated. The tensor is diagonalized to compute a coordinate system that is aligned with the diffusion ellipsoid, a threedimensional shape describing the preferred directions of diffusion in each voxel. The principal axis of the diffusion ellipsoid defines the estimate of the main fiber direction. The lengths of the three ellipsoid axes, or the eigenvalues $\mu$ of the matrix D, define useful properties characterizing the microscopic organization of the tissue. In particular, the apparent diffusion constant (ADC) is an estimate of the diffusion strength, and the fractional anisotropy (FA) quantifies the anisotropy of diffusion. (d) A sagittal FA color map, an axial FA color map, and an axial FA intensity map. FA color maps demonstrate the predominant white matter fiber direction with different colors indicating different directions. Color is composed of red, blue, and green, indexing whether anisotropy is most pronounced from left-to-right, superior-to-inferior, or anterior-to-posterior, respectively. The intensity of the color is proportional to FA: it is large for the hindered diffusion of water, such as in case of myelinated axons, and small for more isotropic regions in the brain, such as gray matter or cerebrospinal fluid. (e) The fiber orientation information of the diffusion tensor eigenvectors and the anisotropy values provide the basis for estimating fiber tracts in the brain. In this example, two seed regions in the internal capsule were placed in the right and the left hemispheres. Fiber estimates originating from these seed regions are rendered in green and blue, respectively. (See Color Plate 31.1 in color plate section.)

DTI provides a subject-specific rendering of fiber tracts, which have a certain intersubject variability independent of variability caused by brain disease, injury, or tumors (Liu et al., 2001; Jellison et al., 2004; Sundgren et al., 2004; Narayana et al., 2007;
Smith et al., 2007). Fiber tracking algorithms usually depend on a number of MRI and analysis parameters, and their results may not be easily comparable between different sites and subjects, although recent progress has been obtained here, 
too (Schwartzman et al., 2005). Figure 1e shows a fiber "tractogram."

In anisotropy maps (Pajevic and Pierpaoli, 1999; Jellison et al., 2004), diffusion anisotropy measures such as FA are used as a proxy for white matter fiber integrity. Figure 1d provides examples of color-coded and gray-scale anisotropy maps. Whereas most conventional MRI contrast mechanisms such as T1, T2, and proton density weighting do not provide quantitative values, diffusion anisotropy maps are derived directly from the quantitative measurements of diffusion in space and thus give us a quantitative means of measuring microscopic tissue properties. In principle, results obtained on different MRI scanners should be comparable. However, due to different scanner hardware, imaging protocols, and data processing algorithms, in practice, most often a direct comparison of numbers obtained on different MRI scanners is not advisable, and corrective measures may be required (Croxson et al., 2005; Johansen-Berg et al., 2005; Farrell et al., 2007; Klein et al., 2007; Landman et al., 2007; Niogi et al., 2007). Fiber tractography and quantitative measures can be combined to quantify diffusion along specific white matter fiber tracts (Jones et al., 2005; Smith et al., 2007). For technical aspects of DTI, and computation of indexes such as FA and ADC, as well as more recent technical advances surpassing the Gaussian (tensor) model of diffusion, see Tournier et al. (2004); Tuch (2004); Alexander (2005); Anderson (2005); Campbell et al. (2005); Hagmann et al. (2006); Kingsley (2006a, b); Minati and Weglarz (2007); Mukherjee et al. (2008b). A recent review of pitfalls of DTI is given by Koch and Norris (2006).

\section{DTI and recovery from brain injury}

There are at least two motivations for the application of DTI in studies of axonal reorganization after brain injury: (i) The sensitivity of DTI with respect to the degree of myelination and axonal density suggests its potential utility as imaging modality to look at axonal reorganization and growth, although still requiring confirmation by accompanying findings of more direct studies using experimental methods (Pons et al.,
1991; Napieralski et al., 1996; Florence et al., 1998; Jain et al., 2000; Carmichael and Chesselet, 2002; Chklovskii et al., 2004; Dancause et al., 2005), or MR spectroscopy (Danielsen et al., 2003). (ii) DTI-based measures such as FA and ADC have proven to provide a sensitive longitudinal measure of white and gray matter changes in the developing and adult brain (McKinstry et al., 2002; Partridge et al., 2004; Bengtsson et al., 2005; deIpolyi et al., 2005; Hermoye et al., 2006; Mukherjee and McKinstry, 2006; Mac Donald et al., 2007; Wozniak and Lim, 2006).

We performed a longitudinal case study of a patient who spontaneously recovered reliable expressive language after 19 years in a minimally conscious state, caused by traumatic brain injury (Voss et al., 2006). Comparison of white matter integrity in the patient with 20 control subjects using ADC and FA identified widespread altered diffusivity and decreased anisotropy. These findings remained unchanged over an 18-month interval between two MRI scans. In addition, we identified large, bilateral regions of posterior white matter with significantly increased anisotropy as measured by FA, which reduced over the same time interval. In contrast, notable increases in anisotropy within the midline cerebellar white matter at the second time point correlated with marked clinical improvements in the patient's motor functions (recovery of limited use of lower extremities and left upper extremity, and improvement in articulation). The finding of changes in the cerebellar white matter was also correlated with an increase in resting metabolism measured by PET in this region. We conjectured that axonal regrowth may account for these observations and provide a biological mechanism for the late recovery of motor function over the interval between DTI measurements. These correlations of altered white matter structures as determined by DTI with clinical improvements suggest the potential clinical utility of longitudinal tracking of patients with brain injuries using DTI.

A more thorough prospective longitudinal DTI study of recovery from severe traumatic brain injury examined the correlation of DTI measurements with clinical outcome variables in 23 subjects (Sidaros et al., 2008). In this study, the 
authors looked in detail at the three principal diffusion tensor components, which enabled them to study the origin of changes, specifically, whether they are more likely to represent axonal injury or demyelination. They found that at the first time point, at 5-11 weeks post-trauma, FA showed widespread reduction in white matter compared to controls. Patients with unfavorable 1-year outcome (measured by the Glasgow Outcome Scale) tended to deviate more from control DTI values than patients with favorable outcome. At the same time, FA in the cerebral peduncle correlated with outcome score at about a year later. After a post-trauma time interval of 9-15 months, follow-up scans showed that FA in patients had increased in the internal capsule and the centrum semiovale. Primarily in patients with favorable outcome, FA reached normal levels or was even higher than normal in these regions. No significant DTI parameter changes over time were found in 14 matched controls during the same time interval. Of note, these increases in FA were restricted to the direction parallel to axonal fibers, an observation interpreted by the investigators as potentially consistent with axonal recovery. The prospective cohort design of this study adds significant evidence in favor of the future use of this technique as a biomarker for prediction of outcomes from traumatic brain injury. Taken together with the longitudinal single subject study of Voss et al. (2006), these findings corroborate the observation of longitudinal changes in DTI during recovery from severe brain injury and add weight to the interpretation that such changes reflect a slow variable of structural change in the brain.

Other studies have shown correlations between recovery scores from severe brain injury and anisotropy values (Han et al., 2007; Skoglund et al., 2008). Ramu et al. (2008) demonstrated significant fiber tract reorganization and regeneration in different parts of the brain in response to spinal cord injury in rats, using histology and DTI. Another study in rats with spinal cord injury investigating the significance of DTI to study neuronal recovery mechanisms, but this time in proximity of the injury, was performed by Deo et al. (2006). The authors found region-specific recovery patterns in the spinal cord white matter, including areas with sustained recovery.

Due to its sensitivity to pathological changes of white matter, DTI has found other widespread clinical applications. For recent reviews of other specific applications, see Lim and Helpern (2002); Sundgren et al. (2004); Johansen-Berg and Behrens (2006); Assaf and Pasternak (2008). DTI sequences are now available from all major clinical MRI scanner manufacturers, along with automated basic postprocessing software, such that they can easily be added to conventional clinical imaging protocols.

\section{Resting state f MRI}

\section{Technique and applications}

In conventional fMRI, fluctuations in the baseline BOLD signal during rest are usually discarded as noise, although they exhibit a rich (but not apparent) spatiotemporal dynamics, as demonstrated by several groups (Biswal et al., 1995; Greicius et al., 2003; Fox et al., 2005; Fox and Raichle, 2007). The most prominent resting state pattern, the "default mode" was originally discovered in resting metabolism positron emission tomography (PET) data (Raichle et al., 2001) and then readily hypothesized to reflect the existence of an organized, baseline default mode of brain function. Interestingly, the default mode network also shows up as the most prominent resting state pattern in BOLD-fMRI data. In addition, the default mode has a direct correlate in cognitive processing. In MRI as well as in PET data, one observes deactivation of areas belonging to the default mode network during attentiondemanding tasks or goal-directed behavior (Raichle et al., 2001; Singh and Fawcett, 2008).

Besides the default mode network, several other resting state networks have been identified more recently (Esposito et al., 2005; De Luca et al., 2006; Mantini et al., 2007). In particular, Mantini et al. found six different functionally meaningful resting state patterns. Figure 2 provides examples for four of them. These six main resting state networks are as follows. 

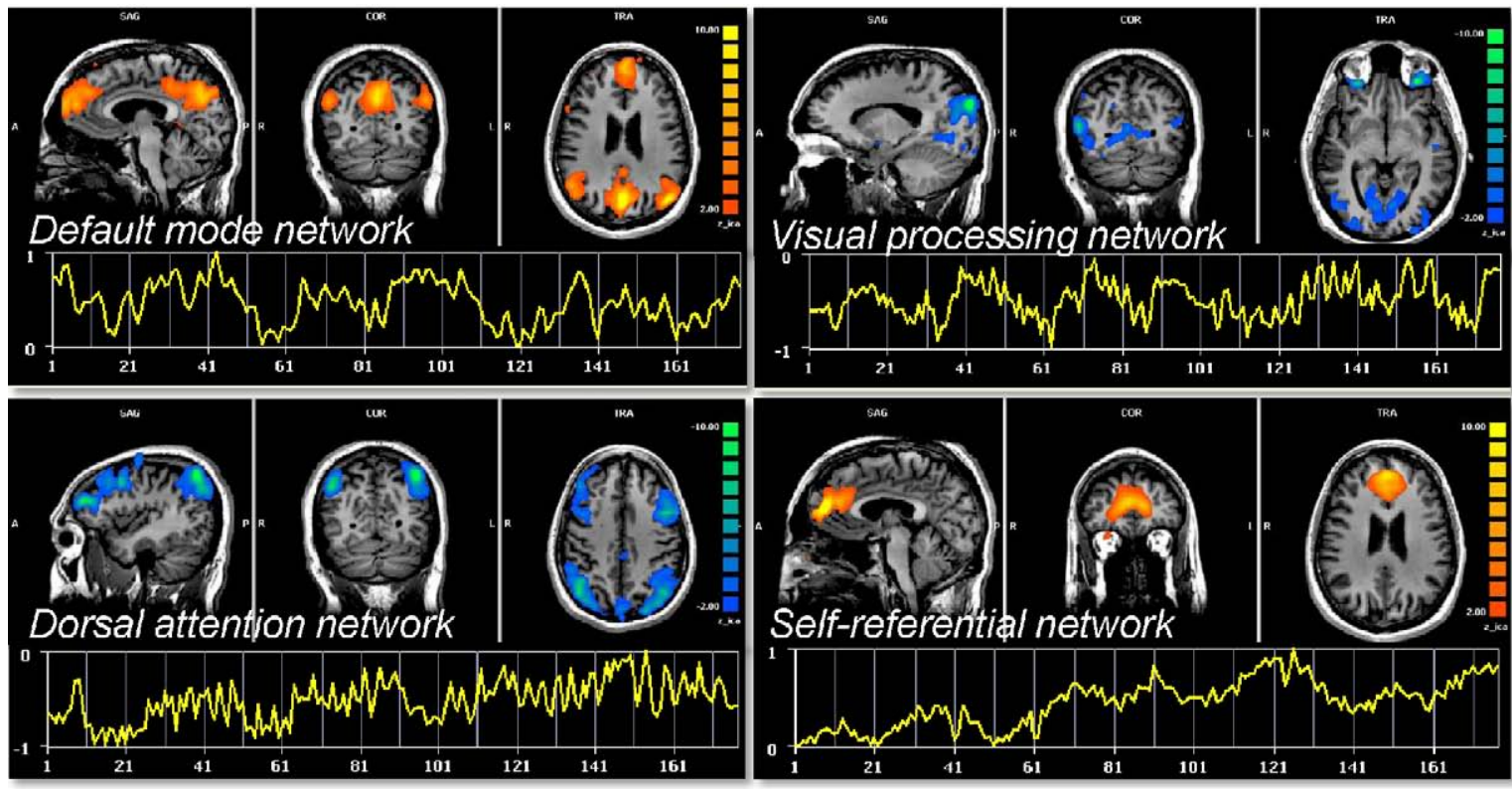

Fig. 2. Four resting state networks in a normal subject. Also shown are corresponding time courses of the independent component coefficients. (Colors quantify the $z$-value of correlation of the time series with the corresponding ICA coefficient. Either positive or negative $z$-values are shown.) (See Color Plate 31.2 in color plate section.)

RSN 1: default mode network, including the posterior cingulate and precuneus, medial prefrontal cortex, dorsal lateral prefrontal cortex, and inferior parietal cortex. RSN 2: dorsal attention network, including the intraparietal sulci, areas at the intersection of precentral and superior frontal sulcus, ventral precentral, and middle frontal gyrus. RSN 3: visual processing network, including retinotopic occipital cortex and temporaloccipital regions. RSN 4: auditory-phonological network, the superior temporal cortices. RSN 5: sensory-motor network, including the precentral, postcentral, and medial frontal gyri, the primary sensory-motor cortices, and the supplementary motor area. RSN 6: self-referential network, including the medial-ventral prefrontal cortex, the pregenual anterior cingulate, the hypothalamus, and the cerebellum. These patterns have considerable overlap with main neuronal networks that have been earlier identified in conventional fMRI studies. (However, the chosen names do not necessarily refer to the exact functions of the resting state networks, which are still putative.) A milestone finding with respect to functional and anatomical significance of resting state networks is the recent demonstration that the oculomotor, the visual, and the somatomotor systems (as determined by corresponding conventional fMRI experiments and fiber staining in anesthetized monkeys) are also identified by BOLD resting state patterns (Vincent et al., 2007).

Recently, it was shown that there is a direct correspondence between fluctuations in EEG oscillations and resting state BOLD baseline fluctuations (Mantini et al., 2007). Correlation of these signals is potentially of considerable relevance as persistent low-frequency oscillations observable in the EEG can provide valuable clinical information about brain function (Niedermeyer and da Silva, 2004; Nunez and Srinivasan, 2005). In further support of the view that the fMRI resting state signal is correlated with electrical activity, Lu et al. (2007) found that in anesthetized rats low-frequency electrical activity underlies the neural correlates of resting state connectivity. This finding is consistent with the suggestion of several authors that resting 
state BOLD fluctuations are representative of synchronous spontaneous fluctuations of largescale networks, which occur at low frequencies (von Stein and Sarnthein, 2000; Schnitzler and Gross, 2005). Furthermore, the fMRI resting state signal differentially registers to specific electrical oscillatory frequency band activity, suggesting that fMRI may be able to distinguish the ongoing from the evoked activity of the brain (Fox and Raichle, 2007). Additional studies have related EEG power fluctuations, particularly within the alpha-band, to resting state patterns (Goldman et al., 2002; Leopold et al., 2003; Laufs et al., 2003; Feige et al., 2005).

These findings make it clear that "resting state" cannot be understood as a complete rest of the brain; it is inevitable that the brain constantly engages in some activity during wakefulness. Rather, a resting state experiment should be seen as an unconstrained experiment as opposed to experiments in which subjects perform specific tasks under defined timing constraints (Lowe et al., 1998). Task-dependent fMRI experiments probe the activity of the brain in certain, usually well-defined areas, whereas resting state activations result from spontaneous, intrinsic fluctuations. The approach of looking at intrinsic fluctuations resembles a methodology from the science of complex systems; for example, by virtue of natural laws such as the fluctuation-dissipation theorem, it is possible to test for the functionality of complex biological and physical systems by analyzing their intrinsic fluctuations (Lauk et al., 1998; Crooker et al., 2004; Rossberg et al., 2004). Resting state fMRI is compatible with the view that the brain largely operates intrinsically, with sensory information only modulating system operations (Burton et al., 2004).

Typical resting state spatial patterns have been computed by "blind" methods such as independent component analysis (ICA) (Formisano et al., 2001; Esposito et al., 2002). The resulting patterns can be classified further as attributable to various sources, such as the BOLD effect, and also to artifacts caused by the experimental procedures or motion. ICA separates spatial components by making the assumption that the spatial network patterns are statistically independent in their time course. In the alternative region of interest (ROI) based approach (Greicius et al., 2003), seed ROIs are selected and signals in them are compared to the signal in other parts of the brain. Statistical measures can be derived to understand the correlation structure of these signals in relation to the seed ROI. Both methods have been shown to be able to define very similar resting state correlation structures (Greicius and Menon, 2004).

\section{Resting state fMRI and potential applications to brain injury}

Within the last 3 years, fMRI resting state network analysis has been applied to a variety of diseases and conditions, such as Alzheimer's Disease (AD) (He et al., 2007; Rombouts et al., 2007; Sorg et al., 2007; Wang et al., 2007), the aging brain (Andrews-Hanna et al., 2007; Wu et al., 2007), blindness (Burton et al., 2004), schizophrenia (Jafri et al., 2008; Liu et al., 2008), attention-deficit/hyperactivity disorder (Zhu et al., 2008; Cao et al., 2006), and epilepsy (Waites et al., 2006; Laufs et al., 2007). In these studies, interesting abnormalities have been found in the resting state network patterns. Recent evidence points toward the possibility of extracting information about functionality and connectivity from resting state fMRI fluctuations (Hampson et al., 2002; Greicius et al., 2003; Seeley et al., 2007). The latter property makes resting state fMRI of particular interest for clinical applications. Often, thalamic structures are involved (De Luca et al., 2006), suggesting that resting state fMRI could develop into a technique to assess global brain network functionality. Conventional fMRI approaches that probe specific functions one-byone by letting the subjects perform specific tasks are technically demanding, and for sufficiently complex tasks, they are usually difficult to perform in the clinical setting. Although such method can be very useful for specific assessments of function (e.g., Owen et al., 2005; Schiff et al., 2005; Giacino et al., 2006), this approach has significant limitations for providing a global view of connectivity in the brain.

The ability to image network integrity or disruptions may be particularly relevant for 
assessments of severely injured brain. For example, in the minimally conscious state (MCS) some patients exhibit behavioral evidence of the recovery of a minimal dynamic network architecture required to organize behavioral sets and to respond to sensory stimuli, not seen in the vegetative state (VS) patients (Giacino et al., 2002). Assessment of functional network activation using fMRI in such patients suggests preservation of large-scale networks that must retain anatomical connectivity (Schiff et al., 2005). While it is expected that normal subjects show similar location and intensity of networks, we hypothesize that subjects with severe focal brain injury may show distortions of the normal patterns. If so, resting state fMRI could eventually provide functional and diagnostic information. However, more experiments and tools are needed in order to better understand the neurophysiological origins of resting state patterns, and to explore the potential utility of resting state fMRI, a diagnostic tool in clinical imaging of the human brain.

\section{a-patient 1}
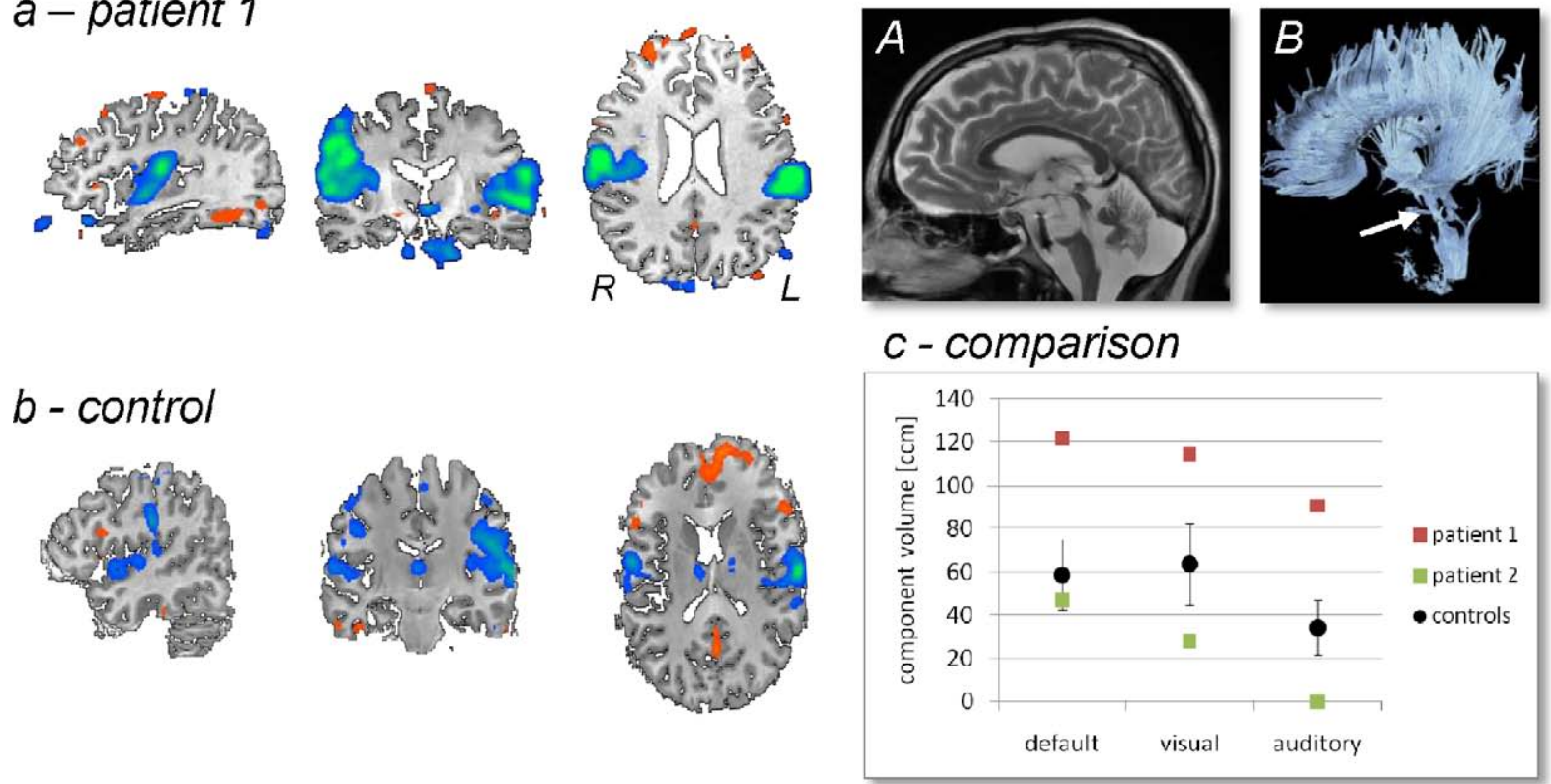

Fig. 3. Auditory-phonological resting state network of (a) Patient 1 and (b) a control subject, as well as (c) a comparison of network volumes between both patients and four control subjects. An anatomical characterization of Patient 1 by means of (A) T1 weighted anatomical imaging and (B) estimates of white matter fiber tracts using DTI. The arrow points to the pontine lesions visible here as disrupted white matter fiber tracts. (See Color Plate 31.3 in color plate section.) 
are also visible in DTI-based white matter fiber tract reconstructions (Fig. 3B). Resting state fluorodeoxyglucose (FDG) PET data showed notable metabolic downregulation of left hemisphere frontotemporal cortical regions (not shown). Patient 2 (female, 56 years) suffered multifocal injuries bilaterally within the anterior and middle cerebral artery distributions following severe cerebral vasospasm induced by anterior communicating artery rupture 32 months prior to MRI.

Resting state networks were identified by independent component analysis (BrainVoyagerQX). The volumes of the RSNs were defined by voxels with $z>2$ in clusters $>1 \mathrm{~cm}^{3}$. Resting metabolism (FDG-PET) of patients was qualitatively compared to volume and connectivity (measured as the maximum $z$-value) of RSN clusters. In all control subjects, we could identify the default mode (RSN 1), visual processing (RSN 3), auditory-phonological (RSN 4), and self-referential (RSN 6) networks.

In Patient 1, we could identify RSN 1, 3, and 4 (Fig. 3a). These RSNs had a significantly larger volume than in the control subjects (Fig. 3c). However, due to the small size of the control group this finding had to be considered preliminary. Metabolic rates (FDG-PET) showed left frontotemporal downregulation, which is traceable to the central thalamic lesion (Van der Werf et al., 1999), and loss of calcarine metabolism on the right, ipsilateral to the LGN infarct. In the fMRI data, the parietal regions of RSN 1 (default) appeared stronger with respect to both volume and connectivity, on the right side. RSN 3 (visual) was smaller on the right side, but had a comparable connectivity. RSN 4 (audio) appeared to be of similar volume and connectivity in both hemispheres. The relatively stronger resting state signal in the right parietal regions of the default mode network may correlate with the metabolic downregulation of the left hemisphere. Similarly, the relatively smaller visual processing network on the right side may correlate with the ipsilateral LGN lesion.

In Patient 2, we could identify the anterior part of RSN 1, as well as RSN 3, 5, and 6. The volume of these networks was reduced but not significantly different from the control subjects (Fig. 3c).
The posterior areas of RSN 1 were missing. This observation was correlated with particularly low metabolism in the corresponding regions, and extensive loss of midline cortical regions.

In conclusion, preliminary studies of alterations in resting state patterns in subjects with brain lesions show correlations with lesion patterns and resting metabolism PET. Further development will be required to assess the potential role of resting state fMRI as an additional tool in evaluating patients with severe brain injuries.

\section{Discussion}

We have reviewed two complementary MRI modalities, DTI and resting state fMRI, to image neuronal structure and function. Whereas DTI has become a mature tool to study various diseases, resting state fMRI is still in its infancy and requires more research to fully understand the observations. However, our two case studies of patients with severe brain lesions provide hints that resting state fMRI may have the potential to become an important tool to assess large-scale connectivity and functional integrity of neuronal networks.

Whether the discussed DTI or resting state fMRI methods, or their combination with other imaging modalities can be used as reliable predictors of outcome after brain lesions remains a challenging problem warranting further research (Calixto et al., 2007; Galanaud et al., 2007; Laureys and Boly, 2007; Schiff, 2007; Rousseau et al., 2008). In particular, more longitudinal, multimodal imaging studies (such as Naganawa et al., 2004; Ewing-Cobbs et al., 2006; Weiss et al., 2007) are required to obtain a clear picture of the predictive value of white matter imaging.

\section{Abbreviations}

$\begin{array}{ll}\text { AD } & \text { Alzheimer's disease } \\ \text { BOLD } & \text { blood-oxygen-level-dependent } \\ \text { DTI } & \text { diffusion tensor imaging } \\ \text { EEG } & \text { electroencephalography } \\ \text { FDG } & \text { fluorodeoxyglucose }\end{array}$




$\begin{array}{ll}\text { fMRI } & \text { functional MRI } \\ \text { MCS } & \text { minimally conscious state } \\ \text { MRI } & \text { magnetic resonance imaging } \\ \text { PET } & \text { positron emission tomography } \\ \text { RSN } & \text { resting state network }\end{array}$

\section{Acknowledgments}

We acknowledge funding from NIH-NICHD, the Dana Foundation, the James S. McDonnell Foundation (NDS), and the Cervical Spine Research Society (HUV).

\section{References}

Alexander, A. L., Lee, J. E., Lazar, M., \& Field, A. S. (2007). Diffusion tensor imaging of the brain. Neurotherapeutics, 4 , 316-329.

Alexander, D. C. (2005). Multiple-fiber reconstruction algorithms for diffusion MRI. Annals of the New York Academy of Sciences, 1064, 113.

Anderson, A. W. (2005). Measurement of fiber orientation distributions using high angular resolution diffusion imaging. Magnetic Resonance in Medicine, 54, 1194-1206.

Andrews-Hanna, J. R., Snyder, A. Z., Vincent, J. L., Lustig, C., Head, D., Raichle, M. E., et al. (2007). Disruption of largescale brain systems in advanced aging. Neuron, 56, 924-935.

Assaf, Y., \& Pasternak, O. (2008). Diffusion tensor imaging (DTI)-based white matter mapping in brain research: a review. Journal of Molecular Neuroscience, 34, 51-61.

Basser, P. J. (1995). Inferring microstructural features and the physiological state of tissues from diffusion-weighted images. NMR in Biomedicine, 8, 333-344.

Basser, P. J., Mattiello, J., \& LeBihan, D. (1994). MR diffusion tensor spectroscopy and imaging. Biophysical Journal, 66, 259-267.

Basser, P. J., \& Pierpaoli, C. (1996). Microstructural and physiological features of tissues elucidated by quantitativediffusion-tensor MRI. Journal of Magnetic Resonance. Series $B, 111,209-219$.

Beaulieu, C., \& Allen, P. S. (1994). Determinants of anisotropic water diffusion in nerves. Magnetic Resonance in Medicine, 31, 394-400.

Bengtsson, S. L., Nagy, Z., Skare, S., Forsman, L., Forssberg, H., \& Ullen, F. (2005). Extensive piano practicing has regionally specific effects on white matter development. Nature Neuroscience, 8, 1148-1150.

Biswal, B., Yetkin, F. Z., Haughton, V. M., \& Hyde, J. S. (1995). Functional connectivity in the motor cortex of resting human brain using echo-planar MRI. Magnetic Resonance in Medicine, 34, 537-541.

Burton, H., Snyder, A. Z., \& Raichle, M. E. (2004). Default brain functionality in blind people. Proceedings of the
National Academy of Sciences of the United States of America, 101, 15500-15505.

Calixto, M., Rafael, R., \& Julius, K. (2007). Will diffusion tensor imaging assess rewiring in PVS' brains? Neurology India, 55, 88 .

Campbell, J. S. W., Siddiqi, K., Rymar, V. V., Sadikot, A. F., \& Pike, G. B. (2005). Flow-based fiber tracking with diffusion tensor and q-ball data: validation and comparison to principal diffusion direction techniques. Neuroimage, 27, 725-736.

Cao, Q. J., Zang, Y. F., Sun, L., Sui, M. Q., Long, X. Y., Zou, Q. H., \& Wang, Y. F. (2006). Abnormal neural activity in children with attention deficit hyperactivity disorder: a resting-state functional magnetic resonance imaging study. Neuroreport, 17, 1033-1036.

Carmichael, S. T., \& Chesselet, M. F. (2002). Synchronous neuronal activity is a signal for axonal sprouting after cortical lesions in the adult. The Journal of Neuroscience, 22, 6062-6070.

Chklovskii, D. B., Mel, B. W., \& Svoboda, K. (2004). Cortical rewiring and information storage. Nature, 431, 782-788.

Coremans, J., Luypaert, R., Verhelle, F., Stadnik, T., \& Osteaux, M. (1994). A method for myelin fiber orientation mapping using diffusion-weighted MR-images. Magnetic Resonance Imaging, 12, 443-454.

Crooker, S. A., Rickel, D. G., Balatsky, A. V., \& Smith, D. L. (2004). Spectroscopy of spontaneous spin noise as a probe of spin dynamics and magnetic resonance. Nature, 431, 49-52.

Croxson, P. L., Johansen-Berg, H., Behrens, T. E. J., Robson, M. D., Pinsk, M. A., Gross, C. G., et al. (2005). Quantitative investigation of connections of the prefrontal cortex in the human and macaque using probabilistic diffusion tractography. The Journal of Neuroscience, 25, 8854-8866.

Dancause, N., Barbay, S., Frost, S. B., Plautz, E. J., Chen, D. F., Zoubina, E. V., et al. (2005). Extensive cortical rewiring after brain injury. The Journal of Neuroscience, 25, 10167-10179.

Danielsen, E. R., Christensen, P. B., rlien-Soborg, P., \& Thomsen, C. (2003). Axonal recovery after severe traumatic brain injury demonstrated in vivo by $1 \mathrm{H}$ MR spectroscopy. Neuroradiology, 45, 722-724.

deIpolyi, A. R., Mukherjee, P., Gill, K., Henry, R. G., Patridge, S. C., Veeraraghavan, S., et al. (2005). Comparing microstructural and macrostructural development of the cerebral cortex in premature newborns: diffusion tensor imaging versus cortical gyration. Neuroimage, 27, 579-586.

De Luca, M., Beckmann, C. F., De Stefano, N., Matthews, P. M., \& Smith, S. M. (2006). fMRI resting state networks define distinct modes of long-distance interactions in the human brain. Neuroimage, 29, 1359-1367.

Deo, A. A., Grill, R. J., Hasan, K. M., \& Narayana, P. A. (2006). In vivo serial diffusion tensor imaging of experimental spinal cord injury. Journal of Neuroscience Research, 83, 801-810.

Douek, P., Turner, R., Pekar, J., Patronas, N., \& LeBihan, D. (1991). MR color mapping of myelin fiber orientation. Journal of Computer Assisted Tomography, 15, 923-929. 
Esposito, F., Formisano, E., Seifritz, E., Goebel, R., Morrone, R., Tedeschi, G., et al. (2002). Spatial independent component analysis of functional MRI time-series: to what extent do results depend on the algorithm used? Human Brain Mapping, 16, 146-157.

Esposito, F., Scarabino, T., Hyvarinen, A., Himberg, J., Formisano, E., Comani, S., et al. (2005). Independent component analysis of fMRI group studies by self-organizing clustering. Neuroimage, 25, 193-205.

Ewing-Cobbs, L., Hasan, K. M., Prasad, M. R., Kramer, L., \& Bachevalier, J. (2006). Corpus callosum diffusion anisotropy correlates with neuropsychological outcomes in twins disconcordant for traumatic brain injury. American Journal of Neuroradiology, 27, 879-881.

Farrell, J. A. D., Landman, B. A., Jones, C. K., Smith, S. A., Prince, J. L., van Zijl, P. C. M., et al. (2007). Effects of signalto-noise ratio on the accuracy and reproducibility of diffusion tensor imaging-derived fractional anisotropy, mean diffusivity, and principal eigenvector measurements at $1.5 \mathrm{~T}$. Journal of Magnetic Resonance Imaging, 26, 756-767.

Feige, B., Scheffler, K., Esposito, F., Di Salle, F., Hennig, J., \& Seifritz, E. (2005). Cortical and subcortical correlates of electroencephalographic alpha rhythm modulation. Journal of Neurophysiology, 93, 2864-2872.

Florence, S. L., Taub, H. B., \& Kaas, J. H. (1998). Large-scale sprouting of cortical connections after peripheral injury in adult macaque monkeys. Science, 282, 1117-1121.

Formisano, E., Esposito, F., Di Salle, F., \& Goebel, R. (2001). Cortex-based independent component analysis of fMRI time-series. Neuroimage, 13, S119.

Fox, M. D., \& Raichle, M. E. (2007). Spontaneous fluctuations in brain activity observed with functional magnetic resonance imaging. Nature Reviews. Neuroscience, 8, 700-711.

Fox, M. D., Snyder, A. Z., Vincent, J. L., Corbetta, M., Van Essen, D. C., \& Raichle, M. E. (2005). The human brain is intrinsically organized into dynamic, anticorrelated functional networks. Proceedings of the National Academy of Sciences of the United States of America, 102, 9673-9678.

Galanaud, D., Naccache, L., \& Puybasset, L. (2007). Exploring impaired consciousness: the MRI approach. Current Opinion in Neurology, 20, 627-631.

Giacino, J. T., Ashwal, S., Childs, N., Cranford, R., Jennett, B., Katz, D. I., et al. (2002). The minimally conscious state definition and diagnostic criteria. Neurology, 58, 349-353.

Giacino, J. T., Hirsch, J., Schiff, N., \& Laureys, S. (2006). Functional neuroimaging applications for assessment and rehabilitation planning in patients with disorders of consciousness. Archives of Physical Medicine and Rehabilitation, 87, S67-S76.

Goldman, R. I., Stern, J. M., Engel, J., \& Cohen, M. S. (2002). Simultaneous EEG and fMRI of the alpha rhythm. Neuroreport, 13, 2487-2492.

Greicius, M. D., Krasnow, B., Reiss, A. L., \& Menon, V. (2003). Functional connectivity in the resting brain: a network analysis of the default mode hypothesis. Proceedings of the National Academy of Sciences of the United States of America, 100, 253-258.
Greicius, M. D., \& Menon, V. (2004). Default-mode activity during a passive sensory task: uncoupled from deactivation but impacting activation. Journal of Cognitive Neuroscience, 16, 1484-1492.

Hagmann, P., Jonasson, L., Maeder, P., Thiran, J. P., Wedeen, V. J., \& Meuli, R. (2006). Understanding diffusion MR imaging techniques: from scalar diffusion-weighted imaging to diffusion tensor imaging and beyond. Radiographics, 26, S205-U219.

Hampson, M., Peterson, B. S., Skudlarski, P., Gatenby, J. C., \& Gore, J. C. (2002). Detection of functional connectivity using temporal correlations in MR images. Human Brain Mapping, 15, 247-262.

Han, B. S., Kim, S. H., Kim, O. L., Cho, S. H., Kim, Y. H., \& Jang, S. H. (2007). Recovery of corticospinal tract with diffuse axonal injury: a diffusion tensor image study. Neurorehabilitation, 22, 151-155.

He, Y., Wang, L., Zang, Y. F., Tian, L. X., Zhang, X. Q., Li, K. C., et al. (2007). Regional coherence changes in the early stages of Alzheimer's disease: a combined structural and resting-state functional MRI study. Neuroimage, $35,488-500$

Hermoye, L., Saint-Maitin, C., Cosnard, G., Lee, S. K., Kim, J., Nassogne, M. C., et al. (2006). Pediatric diffusion tensor imaging: normal database and observation of the white matter maturation in early childhood. Neuroimage, 29, 493-504.

Holodny, A. I., Gor, D. M., Watts, R., Gutin, P. H., \& Ulug, A. M. (2005). Diffusion-tensor MR tractography of somatotopic organization of corticospinal tracts in the internal capsule: initial anatomic results in contradistinction to prior reports. Radiology, 234, 649-653.

Ino, T., Nakai, R., Azuma, T., Yamamoto, T., Tsutsumi, S., \& Fukuyama, H. (2007). Somatotopy of corticospinal tract in the internal capsule shown by functional MRI and diffusion tensor images. Neuroreport, 18, 665-668.

Jafri, M. J., Pearlson, G. D., Stevens, M., \& Calhoun, V. D. (2008). A method for functional network connectivity among spatially independent resting-state components in schizophrenia. Neuroimage, 39, 1666-1681.

Jain, N., Florence, S. L., Qi, H. X., \& Kaas, J. H. (2000). Growth of new brainstem connections in adult monkeys with massive sensory loss. Proceedings of the National Academy of Sciences of the United States of America, 97, 5546-5550.

Jellison, B. J., Field, A. S., Medow, J., Lazar, M., Salamat, M. S., \& Alexander, A. L. (2004). Diffusion tensor imaging of cerebral white matter: a pictorial review of physics, fiber tract anatomy, and tumor imaging patterns. American Journal of Neuroradiology, 25, 356-369.

Johansen-Berg, H., \& Behrens, T. E. J. (2006). Just pretty pictures? What diffusion tractography can add in clinical neuroscience. Current Opinion in Neurology, 19, 379-385.

Johansen-Berg, H., Behrens, T. E. J., Sillery, E., Ciccarelli, O., Thompson, A. J., Smith, S. M., et al. (2005). Functionalanatomical validation and individual variation of diffusion tractography-based segmentation of the human thalamus. Cerebral Cortex, 15, 31-39. 
Jones, D. K., Travis, A. R., Eden, G., Pierpaoli, C., \& Basser, P. J. (2005). PASTA: pointwise assessment of streamline tractography attributes. Magnetic Resonance in Medicine, 53, 1462-1467.

Kim, Y. H., Hong, J. H., Park, C. H., Hua, N., Bickart, K. C., Byun, W. M., et al. (2008). Corticospinal tract location in internal capsule of human brain: diffusion tensor tractography and functional MRI study. Neuroreport, 19, 817-820.

Kingsley, P. B. (2006a). Introduction to diffusion tensor imaging mathematics: part I. Tensors, rotations, and eigenvectors. Concepts in Magnetic Resonance Part A, 28A, 101-122.

Kingsley, P. B. (2006b). Introduction to diffusion tensor imaging mathematics: part II. Anisotropy, diffusionweighting factors, and gradient encoding schemes. Concepts in Magnetic Resonance Part A, 28A, 123-154.

Klein, J. C., Behrens, T. E. J., Robson, M. D., Mackay, C. E., Higham, D. J., \& Johansen-Berg, H. (2007). Connectivitybased parcellation of human cortex using diffusion MRI: establishing reproducibility, validity and observer independence in BA 44/45 and SMA/pre-SMA. Neuroimage, 34, 204-211.

Koch, M. A., \& Norris, D. G. (2006). Artifacts and pitfalls in diffusion MR imaging. In J. Gillard, A. Waldman, \& P. Barker (Eds.), Clinical MR neuroimaging - Diffusion, perfusion and spectroscopy (pp. 99-108). Cambridge: Cambridge University Press.

Landman, B. A., Farrell, J. A. D., Jones, C. K., Smith, S. A., Prince, J. L., \& Mori, S. (2007). Effects of diffusion weighting schemes on the reproducibility of DTI-derived fractional anisotropy, mean diffusivity, and principal eigenvector measurements at $1.5 \mathrm{~T}$. Neuroimage, 36, 1123-1138.

Laufs, H., Hamandi, K., Salek-Haddadi, A., Kleinschmidt, A. K., Duncan, J. S., \& Lemieux, L. (2007). Temporal lobe interictal epileptic discharges affect cerebral activity in "default mode" brain regions. Human Brain Mapping, 28, 1023-1032.

Laufs, H., Krakow, K., Sterzer, P., Eger, E., Beyerle, A., Salek-Haddadi, A., et al. (2003). Electroencephalographic signatures of attentional and cognitive default modes in spontaneous brain activity fluctuations at rest. Proceedings of the National Academy of Sciences of the United States of America, 100, 11053-11058.

Lauk, M., Chow, C. C., Pavlik, A. E., \& Collins, J. J. (1998). Human balance out of equilibrium: nonequilibrium statistical mechanics in posture control. Physical Review Letters, 80, 413-416.

Laureys, S., \& Boly, M. (2007). What is it like to be vegetative or minimally conscious? Current Opinion in Neurology, 20, 609-613.

LeBihan, D., Breton, E., Lallemand, D., Grenier, P., Cabanis, E., \& Lavaljeantet, M. (1986). MR imaging of intravoxel incoherent motions - application to diffusion and perfusion in neurologic disorders. Radiology, 161, 401-407.

Leopold, D. A., Murayama, Y., \& Logothetis, N. K. (2003). Very slow activity fluctuations in monkey visual cortex: implications for functional brain imaging. Cerebral Cortex, 13, 422-433.
Lim, K. O., \& Helpern, J. A. (2002). Neuropsychiatric applications of DTI - a review. NMR in Biomedicine, 15, 587-593.

Liu, Y., Liang, M., Zhou, Y., He, Y., Hao, Y. H., Song, M., et al. (2008). Disrupted small-world networks in schizophrenia. Brain, 131, 945-961.

Liu, W. C., Ollenschleger, M., Kalnin, A., \& Holodny, A. (2001). Two directional method to identify white matter tracts for patients with brain tumors. Neuroimage, 13, S1213.

Lowe, M. J., Mock, B. J., \& Sorenson, J. A. (1998). Functional connectivity in single and multislice echoplanar imaging using resting-state fluctuations. Neuroimage, 7, 119-132.

Lu, H., Zuo, Y., Gu, H., Waltz, J. A., Zhan, W., Scholl, C. A., et al. (2007). Synchronized delta oscillations correlate with the resting-state functional MRI signal. Proceedings of the National Academy of Sciences of the United States of America, 104, 18265-18269.

Mac Donald, C., Dikranian, K., Bayly, P., Holtzman, D., \& Brody, D. (2007). Diffusion tensor imaging reliably detects experimental traumatic axonal injury and indicates approximate time of injury. Journal of Neurotrauma, 24, 1248.

Mantini, D., Perrucci, M. G., Del Gratta, C., Romani, G. L., \& Corbetta, M. (2007). Electrophysiological signatures of resting state networks in the human brain. Proceedings of the National Academy of Sciences of the United States of America, 104, 13170-13175.

McKinstry, R. C., Mathur, A., Miller, J. H., Ozcan, A., Snyder, A. Z., Schefft, G. L., et al. (2002). Radial organization of developing preterm human cerebral cortex revealed by non-invasive water diffusion anisotropy MRI. Cerebral Cortex, 12, 1237-1243.

Minati, L., \& Weglarz, W. P. (2007). Physical foundations, models, and methods of diffusion magnetic resonance imaging of the brain: a review. Concepts in Magnetic Resonance Part A, 30A, 278-307.

Mori, S., \& van Zijl, P. C. M. (2002). Fiber tracking: principles and strategies - a technical review. NMR in Biomedicine, $15,468-480$.

Mori, S., Wakana, S., Nagae-Poetscher, L. M., \& van Zijl, P. C. M. (2005). MRI atlas of human white matter. Amsterdam: Elsevier.

Mukherjee, P., Berman, J. I., Chung, S. W., Hess, C. P., \& Henry, R. G. (2008a). Diffusion tensor MR imaging and fiber tractography: theoretic underpinnings. American Journal of Neuroradiology, 29, 632-641.

Mukherjee, P., Chung, S. W., Berman, J. I., Hess, C. P., \& Henry, R. G. (2008b). Diffusion tensor MR imaging and fiber tractography: technical considerations. American Journal of Neuroradiology, 29, 843-852.

Mukherjee, P., \& McKinstry, R. C. (2006). Diffusion tensor imaging and tractography of human brain development. Neuroimaging Clinics of North America, 16, 19.

Naganawa, S., Sato, C., Ishihra, S., Kumada, H., Ishigaki, T., Miura, S., et al. (2004). Serial evaluation of diffusion tensor brain fiber tracking in a patient with severe diffuse axonal injury. American Journal of Neuroradiology, 25, 1553-1556. 
Napieralski, J. A., Butler, A. K., \& Chesselet, M. F. (1996). Anatomical and functional evidence for lesion-specific sprouting of corticostriatal input in the adult rat. The Journal of Comparative Neurology, 373, 484-497.

Narayana, A., Chang, J., Thakur, S., Huang, W., Karimi, S., Hou, B., et al. (2007). Use of MR spectroscopy and functional imaging in the treatment planning of gliomas. The British Journal of Radiology, 80, 347-354.

Neil, J. J. (1997). Measurement of water motion (apparent diffusion) in biological systems. Concepts in Magnetic Resonance, 9, 385-401.

Niedermeyer, E., \& da Silva, F. L. (Eds). (2004). Electroencephalography: Basic principles, clinical applications, and related fields. Philadelphia: Lippincott Williams \& Wilkins.

Niogi, S. N., Mukherjee, P., \& McCandliss, B. D. (2007). Diffusion tensor imaging segmentation of white matter structures using a Reproducible Objective Quantification Scheme (ROQS). Neuroimage, 35, 166-174.

Nunez, P. L., \& Srinivasan, R. (2005). Electric fields of the brain: The neurophysics of EEG. Oxford: Oxford University Press.

Owen, A. M., Coleman, M. R., Menon, D. K., Johnsrude, I. S., Rodd, J. M., Davis, M. H., et al. (2005). Residual auditory function in persistent vegetative state: a combined PET and fMRI study. Neuropsychological Rehabilitation, 15, 290-306.

Pajevic, S., \& Pierpaoli, C. (1999). Color schemes to represent the orientation of anisotropic tissues from diffusion tensor data: application to white matter fiber tract mapping in the human brain. Magnetic Resonance in Medicine, 42, 526-540.

Park, J. K., Kim, B. S., Choi, G., Kim, S. H., Choi, J. C., \& Khang, H. (2008). Evaluation of the somatotopic organization of corticospinal tracts in the internal capsule and cerebral peduncle: results of diffusion-tensor MR tractography. Korean Journal of Radiology, 9, 191-195.

Partridge, S. C., Mukherjee, P., Henry, R. G., Miller, S. P., Berman, J. I., Jin, H., et al. (2004). Diffusion tensor imaging: serial quantitation of white matter tract maturity in premature newborns. Neuroimage, 22, 1302-1314.

Pons, T. P., Garraghty, P. E., Ommaya, A. K., Kaas, J. H., Taub, E., \& Mishkin, M. (1991). Massive cortical reorganization after sensory deafferentation in adult macaques. Science, 252, 1857-1860.

Raichle, M. E., MacLeod, A. M., Snyder, A. Z., Powers, W. J., Gusnard, D. A., \& Shulman, G. L. (2001). A default mode of brain function. Proceedings of the National Academy of Sciences of the United States of America, 98, 676-682.

Ramu, J., Herrera, J., Grill, R., Bockhorst, T., \& Narayana, P. (2008). Brain fiber tract plasticity in experimental spinal cord injury: diffusion tensor imaging. Experimental Neurology, 212, 100-107.

Rombouts, S. A. R. B., Scheltens, P., Kuijer, J. P. A., \& Barkhof, F. (2007). Whole brain analysis of T2* weighted baseline FMRI signal in dementia. Human Brain Mapping, 28, 1313-1317.

Rossberg, A. G., Bartholome, K., Voss, H. U., \& Timmer, J. (2004). Phase synchronization from noisy univariate signals. Physical Review Letters, 93, 154103.
Rousseau, M. C., Confort-Gouny, S., Catala, A., Graperon, J., Blaya, J., Soulier, E., et al. (2008). A MRS-MRI-fMRI exploration of the brain. Impact of long-lasting persistent vegetative state. Brain Injury, 22, 123-134.

Schiff, N. D. (2007). Bringing neuroimaging tools closer to diagnostic use in the severely injured brain. Brain, 130, 2482-2483.

Schiff, N. D., Rodriguez-Moreno, D., Kamal, A., Kim, K. H. S., Giacino, J. T., Plum, F., et al. (2005). fMRI reveals largescale network activation in minimally conscious patients. Neurology, 64, 514-523.

Schnitzler, A., \& Gross, J. (2005). Normal and pathological oscillatory communication in the brain. Nature Reviews. Neuroscience, 6, 285-296.

Schwartzman, A., Dougherty, R. F., \& Taylor, J. E. (2005). Cross-subject comparison of principal diffusion direction maps. Magnetic Resonance in Medicine, 53, 1423-1431.

Seeley, W. W., Menon, V., Schatzberg, A. F., Keller, J., Glover, G. H., Kenna, H., et al. (2007). Dissociable intrinsic connectivity networks for salience processing and executive control. The Journal of Neuroscience, 27, 2349-2356.

Sidaros, A., Engberg, A., Sidaros, K., Liptrot, M. G., Herning, M., Petersen, P., et al. (2008). Diffusion tensor imaging during recovery from severe traumatic brain injury and relation to clinical outcome: a longitudinal study. Brain, 131, 559-572.

Singh, K. D., \& Fawcett, I. P. (2008). Transient and linearly graded deactivation of the human default-mode network by a visual detection task. Neuroimage, 41, 100-112.

Skoglund, T. S., Nilsson, D., Ljungberg, M., Jonsson, L., \& Rydenhag, B. (2008). Long-term follow-up of a patient with traumatic brain injury using diffusion tensor imaging. Acta Radiologica, 49, 98-100.

Smith, S. M., Johansen-Berg, H., Jenkinson, M., Rueckert, D., Nichols, T. E., Miller, K. L., et al. (2007). Acquisition and voxelwise analysis of multi-subject diffusion data with TractBased Spatial Statistics. Nature Protocols, 2, 499-503.

Sorg, C., Riedl, V., Muhlau, M., Calhoun, V. D., Eichele, T., Laer, L., et al. (2007). Selective changes of resting-state networks in individuals at risk for Alzheimer's disease. Proceedings of the National Academy of Sciences of the United States of America, 104, 18760-18765.

Sundgren, P. C., Dong, Q., Gomez-Hassan, D., Mukherji, S. K., Maly, P., \& Welsh, R. (2004). Diffusion tensor imaging of the brain: review of clinical applications. Neuroradiology, 46, 339-350.

Tournier, J. D., Calamante, F., Gadian, D. G., \& Connelly, A. (2004). Direct estimation of the fiber orientation density function from diffusion-weighted MRI data using spherical deconvolution. Neuroimage, 23, 1176-1185.

Tuch, D. S. (2004). Q-ball imaging. Magnetic Resonance in Medicine, 52, 1358-1372.

Van der Werf, Y. D., Weerts, J. G. E., Jolles, J., Witter, M. P., Lindeboom, J., \& Scheltens, P. (1999). Neuropsychological correlates of a right unilateral lacunar thalamic infarction. Journal of Neurology, Neurosurgery, and Psychiatry, 66, 36-42. 
Vincent, J. L., Patel, G. H., Fox, M. D., Snyder, A. Z., Baker, J. T., Van Essen, D. C., et al. (2007). Intrinsic functional architecture in the anaesthetized monkey brain. Nature, 447, 83-86.

von Stein, A., \& Sarnthein, J. (2000). Different frequencies for different scales of cortical integration: from local gamma to long range alpha/theta synchronization. International Journal of Psychophysiology, 38, 301-313.

Voss, H. U., Ulug, A. M., Dyke, J. P., Watts, R., Kobylarz, E. J., McCandliss, B. D., et al. (2006). Possible axonal regrowth in late recovery from the minimally conscious state. The Journal of Clinical Investigation, 116, 2005-2011.

Waites, A. B., Briellmann, R. S., Saling, M. M., Abbott, D. F., \& Jackson, G. D. (2006). Functional connectivity networks are disrupted in left temporal lobe epilepsy. Annals of Neurology, 59, 335-343

Wang, K., Liang, M., Wang, L., Tian, L. X., Zhang, X. Q., Li, K. C., et al. (2007). Altered functional connectivity in early Alzheimer's disease: a resting-state fMRI study. Human Brain Mapping, 28, 967-978.

Weiss, N., Galanaud, D., Carpentier, A., Naccache, L., \& Puybasset, L. (2007). Clinical review: prognostic value of magnetic resonance imaging in acute brain injury and coma. Critical Care, 11, 230-241.

Wozniak, J. R., \& Lim, K. O. (2006). Advances in white matter imaging: a review of in vivo magnetic resonance methodologies and their applicability to the study of development and aging. Neuroscience and Biobehavioral Reviews, 30, 762-774.

Wu, T., Zang, Y. F., Wang, L., Long, X. Y., Li, K. C., \& Chan, P. (2007). Normal aging decreases regional homogeneity of the motor areas in the resting state. Neuroscience Letters, 423, 189-193.

Zhu, C. Z., Zang, Y. F., Cao, Q. J., Yan, C. G., He, Y., Jiang, T. Z., et al. (2008). Fisher discriminative analysis of resting-state brain function for attention-deficit/hyperactivity disorder. Neuroimage, 40, 110-120. 